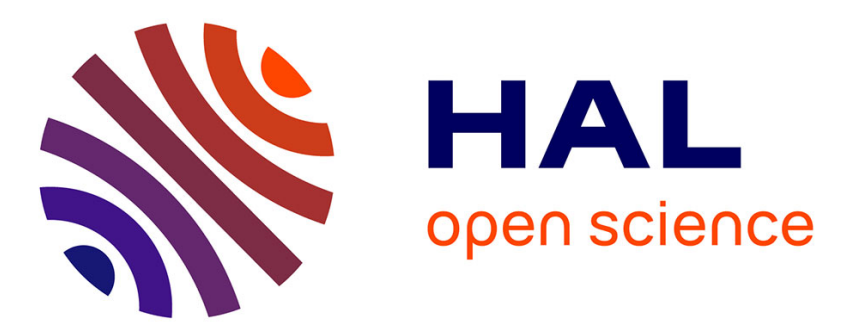

\title{
Direction of Arrival Estimation using EM-ESPRIT with nonuniform arrays
}

Carine El Kassis, José Picheral, Gilles Fleury, Chafic Mokbel

\section{To cite this version:}

Carine El Kassis, José Picheral, Gilles Fleury, Chafic Mokbel. Direction of Arrival Estimation using EM-ESPRIT with nonuniform arrays. Circuits, Systems, and Signal Processing, 2012, 31 (5), pp.17871807. 10.1007/s00034-012-9397-y . hal-00736360

\section{HAL Id: hal-00736360 \\ https://hal-centralesupelec.archives-ouvertes.fr/hal-00736360}

Submitted on 28 Sep 2012

HAL is a multi-disciplinary open access archive for the deposit and dissemination of scientific research documents, whether they are published or not. The documents may come from teaching and research institutions in France or abroad, or from public or private research centers.
L'archive ouverte pluridisciplinaire HAL, est destinée au dépôt et à la diffusion de documents scientifiques de niveau recherche, publiés ou non, émanant des établissements d'enseignement et de recherche français ou étrangers, des laboratoires publics ou privés. 
Noname manuscript No.

(will be inserted by the editor)

\title{
Direction of Arrival Estimation using EM-ESPRIT with nonuniform arrays
}

\author{
Carine El Kassis · José Picheral · Gilles Fleury • \\ Chafic Mokbel
}

Received: date / Accepted: date

\begin{abstract}
This paper deals with the problem of the Direction Of Arrival (DOA) estimation with nonuniform linear arrays. The proposed method is based on the Expectation Maximization method where ESPRIT is used in the maximization step. The key idea is to iteratively interpolate the data to a virtual uniform linear array in order to apply ESPRIT to estimate the DOA. The iterative approach allows to improve the interpolation using the previously estimated DOA. One of this method novelties lies in its capacity of dealing with any nonuniform array geometry. This technique manifests significant performance and computational advantages over previous algorithms such as Spectral MUSIC, EM-IQML and the method based on manifold separation technique. EM-ESPRIT is shown to be more robust to additive noise. Furthermore, EM-ESPRIT fully exploits the advantages of using a nonuniform array over a uniform array: simulations show that for the same aperture and with less number of sensors, the nonuniform array presents almost identical performance as the equivalent uniform array.
\end{abstract}

Keywords Direction of arrival estimation · antenna arrays · nonuniformly spaced arrays · ESPRIT

\section{Introduction}

The estimation of signal parameters from noisy observations is a fundamental problem in array signal processing. The Direction Of Arrival (DOA) estimation is important in many fields including radar, sonar, seismology, communications and medical imaging. Most existing algorithms are devised for Uniform Linear Arrays (ULA). However, the estimation of DOA for Nonuniform Linear Arrays (NLA) is also of great importance in a wide range of applications. For example, sensor failure can lead to missing data problems. This problem occurs for example in seismology

C. El Kassis

Department of Electrical, Computer and Communication Engineering, NDU, Deir El Kamar, Shouf, Lebanon

E-mail: celkassis@ndu.edu.lb

J. Picheral · G. Fleury

Department of Signal Processing and Electronic Systems (SSE), SUPÉLEC, Plateau du moulon, 3, street Joliot-

Curie, 91192 Gif-sur-Yvette Cedex, France

E-mail: firstname.lastname@supelec.fr

C. Mokbel

Faculty of Engineering, University of Balamand, El Koura, Lebanon

E-mail: chafic.mokbel@balamand.edu.lb 
where explosions are used to create the waves. These explosions can deteriorate some of the sensors. Besides, physical and geographical conditions may prohibit the construction of uniformly spaced sensors [1]. Another motivation is to reduce the number of sensors in order to decrease the production cost, minimizing the impact on performance. Furthermore, it would be interesting to be able to increase the aperture of an antenna using the same number of sensors in order to obtain better performance [2] [3].

Among the different geometries of linear arrays, two types are taken into consideration in this paper. The first type is the sparse arrays which can be considered as a ULA where some sensors are omitted and the second type is the nonregular linear arrays where the intersensor separations are chosen in an arbitrary way.

Many works in the literature have studied the estimation of DOA in the NLA case. First of all, the spectral MUSIC [4] algorithm can be directly applied to any NLA geometry. But it presents high computational cost since it implies a multiple search of maximum. Root-MUSIC can only be used in the case of sparse arrays [3] but not for nonregular arrays. Other high resolution methods cannot be directly applied to NLA. Thus, some processing should be applied first in order to use one of the high resolution methods. This processing can be an interpolation of the array output over a Virtual Linear Uniform Array (VULA). The interpolation can be sector-dependant [5], but in this case, the interpolation error is due to the modeling error and cannot be reduced by increasing the Signal-to-Noise Ration (SNR). In [6], the authors propose the Expectation-Maximization (EM) algorithm in order to interpolate the observed data on a VULA using the noise-free model, followed by IQML. This method can be applied only to sparse arrays and presents high computational complexity. Another method is the positive definite Toeplitz completion method [7] which fills the missing values of the Toeplitz covariance matrix of a sparse array which does not present any hole in the coarray function, that means that all the intersensor separations should exist. In the manifold separation technique (MST) [8], the authors proposed to model the NLA steering vector as a product of a matrix that depends only on the array parameters and a Vandermonde vector depending only on the angle. From this Vandermonde structure, a polynomial is obtained, whose roots can be used to estimate the DOA. Reference [9] uses the truncated Fourier series expansion of the Spectral MUSIC criterion which is periodic in order to transform the DOA estimation problem to a polynomial rooting problem. These methods suffer from the error due to the Fourier expansion truncation. In [10], fourth order cumulants are calculated on the data and then ESPRIT [11] or Root-MUSIC [12] can be applied. but this method requires a great number of snapshots in order to obtain a good estimation of the cumulants and it is limited to the case of non Gaussian sources.

In this paper, a new iterative method is proposed: EM-ESPRIT. The principle of [6] is followed but IQML is replaced by ESPRIT to improve the performance and reduce the computational complexity in comparison to IQML. Furthermore, this method is extended to the case of nonregular arrays. This method presents better performance than most of the existing ones. The EM algorithm is used to interpolate the NLA outputs on a Virtual Uniform Linear Array (VULA) using the signal model. Since the new constructed data form a uniform array, a high resolution method can be directly applied to estimate the DOA. ESPRIT [11] is chosen because it is a simple and efficient method. The iterative approach allows to improve the interpolation using the previously estimated DOA. Since the interpolation is based on the signal model, the error due to interpolation is reduced iteratively. Thus, EM-ESPRIT gives better results than the interpolation of Friedlander [5] specially in the asymptotic domain. Furthermore, no error due to truncation of the Fourier expansion is introduced as in [8] and [9], thus, EM-ESPRIT behaves better than these two methods. The superiority of EM-ESPRIT over other methods, specially on noisy data with low SNR, is illustrated by simulations in this paper. The originality of EMESPRIT is that it can be applied to any type of NLA: the sparse array and the nonregular array, 
yielding best performance for both types. Besides, EM-ESPRIT exploits the advantages of using an NLA instead of a ULA. This advantage is illustrated by simulation in this paper. In particular, the reduction of a certain number of sensors of a uniform array degrades only very slightly the performance with EM-ESPRIT, thus the production and calculation costs are reduced compared to ULA.

This paper is organized as follows. Section 2 introduces the signal model used along the paper. In section 3, the complete data construction, the EM general approach and the EM-ESPRIT are presented in the case of sparse arrays. The method is extended to the case of nonregular linear arrays in section 4. The computational complexity of EM-ESPRIT is given in section 5 . Simulation results are presented in section 6 to support the analysis and section 7 provides some conclusions.

\section{Signal model}

Consider $N$ far-field narrowband sources incident on an $M$-elements linear array, $(M \geq N)$, from directions $\boldsymbol{\theta}=\left[\theta_{1}, \ldots, \theta_{N}\right]^{\top}$. The sensors, assumed to be omnidirectional, are situated at positions $d_{m}(m=1, \ldots, M)$. Two kinds of NLA are considered: the sparse array, which can be considered as a ULA where some elements are omitted, i.e. $d_{m}=k_{m} \Delta$ where $\Delta$ is the ULA intersensor separation and $k_{m}$ is an integer, and the nonregular linear array, where the sensors positions are chosen in an arbitrary way.

Grouping the signals received by the $M$ array elements in the $M \times 1$ vector $\mathbf{y}(t)$, the sensor outputs can be written as:

$$
\mathbf{y}(t)=\mathbf{A}(\boldsymbol{\theta}) \mathbf{s}(t)+\boldsymbol{\nu}(t),
$$

where $\mathbf{A}(\boldsymbol{\theta})=\left[\mathbf{a}\left(\theta_{1}\right), \ldots, \mathbf{a}\left(\theta_{N}\right)\right]$ is the $M \times N$ steering matrix and $\mathbf{a}\left(\theta_{n}\right)$ is the steering vector of the $n$-th source:

$$
\mathbf{a}\left(\theta_{n}\right)=\left[e^{-j 2 \pi \frac{d_{1} \sin \theta_{n}}{\lambda}}, \ldots, e^{-j 2 \pi \frac{d_{M} \sin \theta_{n}}{\lambda}}\right]^{\top} .
$$

The $N \times 1$ vector $\mathbf{s}(t)$ contains the complex amplitude of the source signals. The $M \times 1$ vector $\boldsymbol{\nu}(t)$ represents a complex additive white Gaussian noise, with zero mean and such as $E\left\{\boldsymbol{\nu}(t) \boldsymbol{\nu}(t)^{H}\right\}=$ $\sigma^{2} \mathbf{I}$. the sources are assumed to be independent.

The received signal is sampled, and the received data $\mathbf{Y}=\left[\mathbf{y}\left(t_{1}\right), \ldots, \mathbf{y}\left(t_{L}\right)\right]$ can be expressed as:

$$
\mathbf{Y}=\mathbf{A}(\boldsymbol{\theta}) \mathbf{S}+\mathbf{V},
$$

where $L$ is the number of samples, $\mathbf{S}=\left[\mathbf{s}\left(t_{1}\right), \ldots, \mathbf{s}\left(t_{L}\right)\right]$ and $\mathbf{V}=\left[\boldsymbol{\nu}\left(t_{1}\right), \ldots, \boldsymbol{\nu}\left(t_{L}\right)\right]$.

The parameters to estimate are the DOA $\boldsymbol{\theta}=\left[\theta_{1}, \ldots, \theta_{N}\right]^{T}$. The number of sources $N$ is considered as known.

\section{DOA estimation in the case of sparse arrays}

In this section, the method is presented in the case of sparse array and in the next section it is generalized to the case of nonregular arrays. Consider the NLA of $M$ sensors having an aperture of $M^{\prime}\left(M^{\prime} \geq M\right)$ that can be seen as a ULA of $M^{\prime}$ sensors where some sensors are omitted. Thus, consider the VULA of $M^{\prime}$ sensors formed by the NLA sensors and by the omitted virtual sensors as represented in Fig. 1. In this case, the nonuniform array output can be seen as incomplete data and the complete data are the output of the VULA. Thus, the EM algorithm approach can be applied in order to estimate the complete data and the model parameters $\boldsymbol{\theta}$. In summary, The main idea consists in interpolating the nonuniform array in a way that a uniform array is formed and then estimating the DOA through ESPRIT [11] applied to the new VULA. 


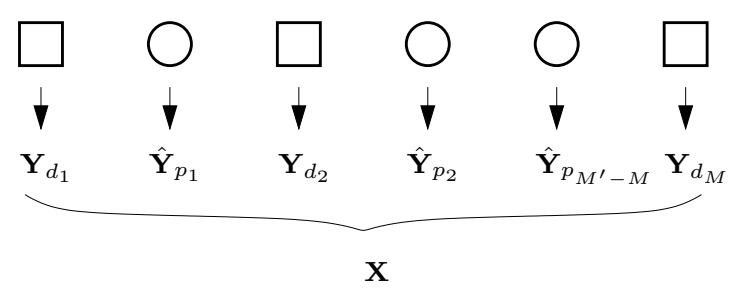

Fig. 1 Example of NLA and its equivalent VULA, with $\mathbf{d}=[0,2,5] \frac{\lambda}{2}, \mathbf{p}=[1,3,4] \frac{\lambda}{2}$ and $\mathbf{g}=[1,0,1,0,0,1]$. $\square$ : existing sensors $(\mathbf{Y})$, O: omitted sensors $\left(\hat{\mathbf{Y}}_{\mathbf{p}}\right)$.

\subsection{Data construction}

Let $\mathbf{Y}$ denote the observed data (or incomplete data) corresponding to the $M$ sensors output of the NLA output and $\mathbf{X}$ the complete data that correspond to the $M^{\prime}$ virtual sensors output of the VULA output. $\mathbf{X}$ is formed by the observed data and the missing data corresponding to the missing sensors output denoted by $\mathbf{Y}_{\mathbf{p}}$. The relation between $\mathbf{Y}$ and $\mathbf{X}$ can be described as a linear transformation mapping $\mathbf{X}$ to $\mathbf{Y}$ given by:

$$
\mathbf{Y}=\mathbf{G}^{\top} \mathbf{X}
$$

where the $M^{\prime} \times M$ matrix $\mathbf{G}$ is constructed by eliminating the zero columns of $\operatorname{diag}(\mathbf{g})$. The $M^{\prime} \times 1$ vector $\mathbf{g}$ describes the binary transformation between $\mathbf{X}$ and $\mathbf{Y}$ : the $m$-th component of $\mathrm{g}$ is 1 if the $m$-th sensor of the VULA is part of the NLA and 0 otherwise. For example in Fig. 1, since the sensors $0,2,5$ of the VULA form the NLA, $\mathbf{g}=[1,0,1,0,0,1]$. The relation between the output of the missing sensors $\mathbf{Y}_{\mathbf{p}}$ and the complete data $\mathbf{X}$ is expressed by:

$$
\mathbf{Y}_{\mathbf{p}}=\overline{\mathbf{G}}^{\top} \mathbf{X}
$$

where $\overline{\mathbf{G}}$ is of dimension $M^{\prime} \times\left(M^{\prime}-M\right)$. It is constructed similarly to $\mathbf{G}$.

By construction, the noise free parametric model of the complete data $\mathbf{X}$ is given by:

$$
\boldsymbol{\mu}(\boldsymbol{\Theta})=\left(\mathbf{G A}(\boldsymbol{\theta})+\overline{\mathbf{G}} \mathbf{A}_{\mathbf{p}}(\boldsymbol{\theta})\right) \mathbf{S}
$$

where $\boldsymbol{\Theta}=\left[\boldsymbol{\theta}^{\top}, \operatorname{vect}(S)^{\top}\right]$ are the source parameters to be estimated.

$\mathbf{A}_{\mathbf{p}}(\boldsymbol{\theta})$ is the steering matrix for the omitted sensors. Let the vector $\mathbf{p}=\left[p_{1}, \ldots, p_{M^{\prime}-M}\right]$ of length $\left(M^{\prime}-M\right)$ represent the positions of the omitted sensors. Then, $\mathbf{A}_{\mathbf{p}}(\boldsymbol{\theta})$ can be written as:

$$
\mathbf{A}_{\mathbf{p}}(\boldsymbol{\theta})=\left[\mathbf{a}_{\mathbf{p}}\left(\theta_{1}\right), \ldots, \mathbf{a}_{\mathbf{p}}\left(\theta_{N}\right)\right], \quad \mathbf{a}_{\mathbf{p}}\left(\theta_{n}\right)=\left[e^{-j 2 \pi \frac{p_{1} \sin \theta_{n}}{\lambda}}, \ldots, e^{-j 2 \pi \frac{p_{M^{\prime}-M} \sin \theta_{n}}{\lambda}}\right]^{\top}
$$

Notice that the model $\boldsymbol{\mu}(\boldsymbol{\Theta})$ can be rewritten as

$$
\boldsymbol{\mu}(\boldsymbol{\Theta})=\mathbf{A}_{V U L A}(\boldsymbol{\theta}) \mathbf{S},
$$

where $\mathbf{A}_{V U L A}(\boldsymbol{\theta})=\mathbf{G A}(\boldsymbol{\theta})+\overline{\mathbf{G}} \mathbf{A}_{\mathbf{p}}(\boldsymbol{\theta})$ is the steering matrix of a uniform linear array, in this case, the VULA. 


\subsection{The EM general approach}

Basically, EM is an iterative approach of the maximum likelihood estimator (ML) [13]. Each iteration is composed of two steps: the Expectation step (E-step) and the Maximization step $\left(M\right.$-step). The E-step is the estimation of the conditional likelihood of the complete data $\hat{\mathbf{X}}^{(k)}$, given the incomplete data $\mathbf{Y}$ and the previous estimate of the parameters $\hat{\boldsymbol{\Theta}}^{(k-1)}$, where $k$ is the iteration number. In the $M$-step, the parameters $\hat{\boldsymbol{\Theta}}^{(k)}$ are estimated by maximizing the conditional likelihood criterion. Let $L(\Theta)$, the log-likelihood of the observed data, be the function to be maximized. It can be shown that $L(\boldsymbol{\Theta})$ increases at each iteration and that $\hat{\boldsymbol{\Theta}}^{(k)}$ converges to a local optimum as solution to the ML (see for instance [13]).

A justification of the EM algorithm is as follows. Using Bayes' rule, and taking the logarithm of the probability densities,

$$
\ln f_{\mathbf{y}}(\mathbf{Y} \mid \boldsymbol{\Theta})=\ln f_{\mathbf{X}}(\mathbf{X} \mid \boldsymbol{\Theta})-\ln f_{\mathbf{x} \mid \mathbf{Y}}(\mathbf{X} \mid \mathbf{Y}, \boldsymbol{\Theta})
$$

Taking the conditional expectation of (9) given $\mathbf{Y}$ at the parameter value $\hat{\boldsymbol{\Theta}}^{(k-1)}$ yields

$$
L(\boldsymbol{\Theta}) \triangleq \ln f_{\mathbf{y}}(\mathbf{Y} \mid \boldsymbol{\Theta})=U\left(\boldsymbol{\Theta} \mid \hat{\boldsymbol{\Theta}}^{(k-1)}\right)-V\left(\boldsymbol{\Theta} \mid \hat{\boldsymbol{\Theta}}^{(k-1)}\right),
$$

where

$$
\begin{aligned}
& U\left(\boldsymbol{\Theta} \mid \hat{\boldsymbol{\Theta}}^{(k-1)}\right)=E\left\{\ln \left\{f_{\mathbf{X}}(\mathbf{X} \mid \boldsymbol{\Theta})\right\} \mid \mathbf{Y}, \hat{\boldsymbol{\Theta}}^{(k-1)}\right\}, \\
& V\left(\boldsymbol{\Theta} \mid \hat{\boldsymbol{\Theta}}^{(k-1)}\right)=E\left\{\ln \left\{f_{\mathbf{x} \mid \mathbf{Y}}(\mathbf{X} \mid \mathbf{Y}, \boldsymbol{\Theta})\right\} \mid \mathbf{Y}, \hat{\boldsymbol{\Theta}}^{(k-1)}\right\} .
\end{aligned}
$$

Knowing that $V\left(\boldsymbol{\Theta} \mid \hat{\boldsymbol{\Theta}}^{(k-1)}\right) \leq V\left(\hat{\boldsymbol{\Theta}}^{(k-1)} \mid \hat{\boldsymbol{\Theta}}^{(k-1)}\right)$ (Jensen's inequality), if $U\left(\boldsymbol{\Theta} \mid \hat{\boldsymbol{\Theta}}^{(k-1)}\right)>$ $U\left(\hat{\boldsymbol{\Theta}}^{(k-1)} \mid \hat{\boldsymbol{\Theta}}^{(k-1)}\right)$, then $L(\boldsymbol{\Theta})>L\left(\hat{\boldsymbol{\Theta}}^{(k-1)}\right)$. Thus, the maximization of $U\left(\boldsymbol{\Theta} \mid \hat{\boldsymbol{\Theta}}^{(k-1)}\right)$ improves the likelihood function. Exploiting this property, the EM algorithm can be described as follows.

It starts with an initial guess $\hat{\boldsymbol{\Theta}}^{(0)}$, and maximizes $L(\boldsymbol{\Theta})$ by iterating the $\mathrm{E}$ and M-steps, i.e. at iteration $k$ :

E-step: Compute $U\left(\boldsymbol{\Theta} \mid \hat{\boldsymbol{\Theta}}^{(k-1)}\right)$.

M-step: Estimate $\hat{\boldsymbol{\Theta}}^{(k)}$ as $\hat{\boldsymbol{\Theta}}^{(k)}=\arg \max _{\boldsymbol{\Theta}} U\left(\boldsymbol{\Theta} \mid \hat{\boldsymbol{\Theta}}^{(k-1)}\right)$.

\subsection{EM-ESPRIT}

In this paragraph, a new method for DOA estimation is proposed in the case of sparse arrays. Using the formalism established above, it can be shown that the E-step is equivalent to the estimation of the missing sensors output using an interpolation based on the signal model. In the $M$-step, in order to reduce the complexity of the maximization of the ML criterion, the maximization of $U\left(\boldsymbol{\Theta} \mid \hat{\boldsymbol{\Theta}}^{(k-1)}\right)$ is replaced by the high resolution method ESPRIT, since the virtual array is uniform. While the E-step of the proposed approach follows exactly the classical EM algorithm, ESPRIT [11] used in the $M$-step is sub-optimal compared to the maximization of the likelihood function. Nevertheless, it is known that ESPRIT provides a close estimation to the ML [14]. Now, EM is applied in the NLA case in order to estimate the DOA. 


\subsubsection{E-step}

The application of the general algorithm involves the determination of $U\left(\boldsymbol{\Theta} \mid \hat{\boldsymbol{\Theta}}^{(k-1)}\right) \cdot \boldsymbol{\mu}(\boldsymbol{\Theta})$ is the unknown mean of the matrix $\mathbf{X}$ which is Gaussian and its covariance matrix equals $\sigma^{2} \mathbf{I}$. Thus, $f_{\mathbf{x}}(\mathbf{X} \mid \boldsymbol{\Theta})$, the probability density function of $\mathbf{X}$ given $\boldsymbol{\Theta}$, is given by:

$$
\ln f_{\mathbf{X}}(\mathbf{X} \mid \boldsymbol{\Theta})=-M^{\prime} L \ln \left(\pi \sigma^{2}\right)-\|\mathbf{X}-\boldsymbol{\mu}(\boldsymbol{\Theta})\|^{2} / \sigma^{2}
$$

Without getting into details, $U\left(\boldsymbol{\Theta} \mid \hat{\boldsymbol{\Theta}}^{(k-1)}\right)$ can be written as:

$$
U\left(\boldsymbol{\Theta} \mid \hat{\boldsymbol{\Theta}}^{(k-1)}\right)=c-\left\|\hat{\mathbf{X}}^{(k)}-\boldsymbol{\mu}\left(\hat{\boldsymbol{\Theta}}^{(k-1)}\right)\right\|^{2} / \sigma^{2}
$$

where $c$ is a constant independent of $\boldsymbol{\Theta}$ and $\hat{\mathbf{X}}^{(k)}$ is the expectation of $\mathbf{X}$ given $\mathbf{Y}$ and $\hat{\boldsymbol{\Theta}}^{(k-1)}$ :

$$
\hat{\mathbf{X}}^{(k)}=E\left\{\mathbf{X} \mid \mathbf{Y} ; \hat{\boldsymbol{\Theta}}^{(k-1)}\right\} \text {. }
$$

From (12), the maximization of $U\left(\boldsymbol{\Theta} \mid \hat{\boldsymbol{\Theta}}^{(k-1)}\right)$ reduces to the minimization of $\left\|\hat{\mathbf{X}}^{(k)}-\boldsymbol{\mu}(\boldsymbol{\Theta})\right\|^{2}$. Thus $\hat{\mathbf{X}}^{(k)}$ is the only parameter to estimate in the E-step. The expression of $\hat{\mathbf{X}}^{(k)}$ is proved [15] to be equal to:

$$
\hat{\mathbf{X}}^{(k)}=\boldsymbol{\mu}\left(\hat{\boldsymbol{\Theta}}^{(k-1)}\right)+\mathbf{G}^{*}\left[\mathbf{G}^{\top} \mathbf{G}^{*}\right]^{-1}\left(\mathbf{Y}-\mathbf{G}^{\top} \boldsymbol{\mu}\left(\hat{\boldsymbol{\Theta}}^{(k-1)}\right)\right)
$$

Replacing $\boldsymbol{\mu}\left(\hat{\boldsymbol{\Theta}}^{(k-1)}\right)$ by its expression and making the required calculation, (14) becomes:

$$
\hat{\mathbf{X}}^{(k)}=\mathbf{G Y}+\overline{\mathbf{G}} \mathbf{A}_{\mathbf{p}}\left(\hat{\boldsymbol{\theta}}^{(k-1)}\right) \hat{\mathbf{S}}^{(k-1)},
$$

where $\hat{\mathbf{S}}^{(k)}$ is estimated by

$$
\hat{\mathbf{S}}^{(k)}=\mathbf{A}^{\dagger}\left(\hat{\boldsymbol{\theta}}^{(k)}\right) \mathbf{Y}
$$

and $\mathbf{A}^{\dagger}=\left(\mathbf{A}^{H} \mathbf{A}\right)^{-1} \mathbf{A}^{H}$ represents the pseudo-inverse of $\mathbf{A}$ and $\mathbf{A}^{H}$ is the hermitian of $\mathbf{A}$. Following (15), the estimates of the complete data $\hat{\mathbf{X}}^{(k)}$ are equal to the measured data $\mathbf{Y}$ for the rows corresponding to the existing sensors, and for the rows of the missing data, the data are interpolated using the parametric model $\boldsymbol{\mu}\left(\hat{\boldsymbol{\Theta}}^{(k-1)}\right)$. Let $\hat{\mathbf{Y}}_{\mathbf{p}}^{(k)}=\mathbf{A}_{\mathbf{p}}\left(\hat{\boldsymbol{\theta}}^{(k-1)}\right) \hat{\mathbf{S}}^{(k-1)}$ be the interpolated missing data. Notice that the noise contribution in $\hat{\mathbf{Y}}_{\mathbf{p}}^{(k)}$ could be omitted, because its expectation is null.

\subsubsection{M-step}

Since the new virtual array is uniform, any conventional method for DOA estimation in the case of uniform arrays can be used at one condition: it should at least ensure to increase the likelihood function. The ESPRIT algorithm is chosen in order to estimate the $\hat{\boldsymbol{\Theta}}^{(k)}$ since it presents good performance and low complexity. The ESPRIT algorithm is based on the eigendecomposition of the covariance matrix. Thus the number of sources $N$ should be smaller than $M$, otherwise, it is known that the weakest sources cannot be estimated [11]. The VULA data correlation matrix, $\hat{\mathbf{R}}^{(k)}$, is estimated using $\hat{\mathbf{X}}^{(k)}$ and the noise contribution on the interpolated data is modeled by $\overline{\mathbf{G}} \overline{\mathbf{G}}^{H} \hat{\sigma}^{2}$, assuming that the noise contribution in $\hat{\mathbf{Y}}_{\mathbf{p}}^{(k)}$ has the same statistical properties as $\boldsymbol{\nu}(t)$ :

$$
\hat{\mathbf{R}}^{(k)}=\frac{1}{L} \hat{\mathbf{X}}^{(k)} \hat{\mathbf{X}}^{(k) H}+\hat{\sigma}^{2} \overline{\mathbf{G}} \overline{\mathbf{G}}^{H},
$$

where $\hat{\sigma}^{2}$ is a consistent estimate of $\sigma^{2}$ obtained by averaging the smallest eigenvalues of the $\mathbf{Y}$ covariance matrix. The $\hat{\boldsymbol{\Theta}}^{(k)}$ are estimated by ESPRIT which exploits the translational invariance structure of the covariance data of the VULA, $\hat{\mathbf{R}}^{(k)}$. 


\subsection{Summary of EM-ESPRIT}

In this section, the proposed method EM-ESPRIT is summarized in an algorithmic form describing the calculation steps. This method is iterative.

1. Initialization: $k=0$, choose $\hat{\boldsymbol{\theta}}^{(0)}$ and estimate the noise variance $\hat{\sigma}^{2}$.

2. Estimate the complete data $\hat{\mathbf{X}}^{(k)}$ using (15) and estimate the covariance matrix $\hat{\mathbf{R}}^{(k)}$ using (17).

3. Use ESPRIT to estimate $\hat{\boldsymbol{\theta}}^{(k)}$.

4. Check convergence of $\hat{\boldsymbol{\theta}}^{(k)}$. If not, $k=k+1$, go to step 2 .

The convergence test is expressed by:

$$
\left\|\hat{\boldsymbol{\theta}}^{(k)}-\hat{\boldsymbol{\theta}}^{(k-1)}\right\| \leq \epsilon
$$

where $\epsilon$ is chosen following the precision constraints required by the application.

\section{DOA estimation in the case of nonregular arrays}

In the nonregular arrays, the intersensor separations are not a multiple of $\Delta$. The sensors are placed in an arbitrary way. Consider the nonregular array of $M$ sensors represented at Fig. 2.

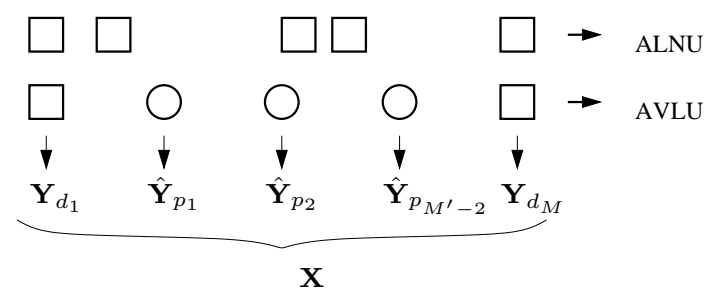

Fig. 2 Example of a nonregular array and its equivalent VULA, with $\mathbf{g}=[1,0,0,0,1]$. In the VULA, $\square$ : existing sensors $(\mathbf{Y})$, O: omitted sensors $\left(\hat{\mathbf{Y}}_{\mathbf{p}}\right)$.

The nonregular arrays are used when the construction of a uniform array is not possible due to physical constraints. EM-ESPRIT is generalized to the case of nonregular arrays in this section. Thus, one of the advantages of the proposed method is its ability to estimate the DOA for any geometry type of an NLA. In order to use the proposed method to the case of nonregular arrays, some modifications are applied to the steps of section 3 .

\subsection{Data construction}

The sensors of the nonregular array cannot be a part of the VULA sensors and the existing sensors cannot be simply completed in order to form the complete data. Nevertheless, the idea of VULA should be kept to be able to apply ESPRIT. The VULA is formed of $M^{\prime}$ sensors, has the same aperture as the nonregular array so that the sensors at each edge of the two arrays coincide. The output of the remaining $\left(M^{\prime}-2\right)$ sensors are interpolated. The construction of the VULA is represented at Fig. 2. The VULA has the same aperture as the nonregular array but the number of sensors $M^{\prime}$ is not necessarily equal to $M$. The effect of the choice of $M^{\prime}$ is shown later: $M^{\prime}=M$ gives the best results provided that it guarantees an intersensor separation 
$\Delta<\frac{\lambda}{2}$. The change of the VULA construction leads to a modification in the construction of the complete data $\mathbf{X}$. Since only the first and last sensors are taken into consideration in the VULA, the vector $\mathbf{g}$ of dimension $M^{\prime}$ has only the first and last elements equal to 1 and the other elements are set to zero: $\mathbf{g}=[1,0, \ldots, 0,1]$. The matrix $\mathbf{G}$ is of dimension $M^{\prime} \times 2$, the vector of omitted sensors is equal to $\mathbf{p}=\left[p_{1}, \ldots, p_{M^{\prime}-2}\right]$ and the matrix $\overline{\mathbf{G}}$ has the dimension $M^{\prime} \times\left(M^{\prime}-2\right)$.

\subsection{EM-ESPRIT}

The noiseless parametric model $\mathbf{X}$ is still given by (6). Besides, the expression of $\ln f_{\mathbf{x}}(\mathbf{X} \mid \boldsymbol{\Theta})$ does not change and the DOA are estimated by maximizing $U\left(\boldsymbol{\Theta} \mid \hat{\boldsymbol{\Theta}}^{(k-1)}\right)$.

In the E-step, the algorithm does not change. The same procedure is applied since the statistical properties of the complete data did not change. Thus, $\hat{\mathbf{X}}^{(k)}=E\left\{\mathbf{X} \mid \mathbf{Y} ; \hat{\boldsymbol{\Theta}}^{(k-1)}\right\}$ should be computed using eq. (15). The complete data $\hat{\mathbf{X}}^{(k)}$ is equal to the measured data $\mathbf{Y}$ for the rows corresponding to the sensors at each end of the array and for the rows of the missing data, the data are interpolated using the parametric model $\boldsymbol{\mu}(\boldsymbol{\Theta})$ :

$$
\hat{\mathbf{Y}}_{\mathbf{p}}^{(k)}=\mathbf{A}_{\mathbf{p}}\left(\hat{\boldsymbol{\theta}}^{(k-1)}\right) \hat{\mathbf{S}}^{(k-1)}
$$

where $\hat{\mathbf{S}}^{(k)}=\mathbf{A}^{\dagger}\left(\hat{\boldsymbol{\theta}}^{(k)}\right) \mathbf{Y}$, same expression of eq. (16). Notice that even if the first and the last sensors are the only ones taken into consideration in the construction of the VULA, all the observed data $\mathbf{Y}$ are used in the interpolation. These data appear in the calculation of $\hat{\mathbf{S}}^{(k)}$ which is estimated using $\mathbf{Y}$, as shown in eq. (16).

The $M$-step is identical to the one explained in paragraph 3.3. The ESPRIT algorithm is applied on the VULA in order to estimate the DOA. In conclusion, the same algorithm summarized in section 3.4 is applied in the case of nonregular arrays but with a modification in the construction of $\mathbf{X}$.

\section{Computational complexity of EM-ESPRIT}

In this section, the computational complexity of the main steps of EM-ESPRIT is evaluated. Recall that $M$ is the number of sensors in the NLA, $M^{\prime}$ the number of sensors in the VULA, $N$ the sources number and $L$ the number of snapshots. Operation means any mathematical operation carried out during the process, whether it is multiplication or addition. Consider at first the case of one iteration, the complexity of the calculation of $\hat{\mathbf{S}}^{(k)}=\mathbf{A}^{\dagger}\left(\hat{\boldsymbol{\theta}}^{(k)}\right) \mathbf{Y}$ is $(2 M N L)$; besides, $\mathbf{A}^{\dagger}\left(\hat{\boldsymbol{\theta}}^{(k)}\right)=\left(\mathbf{A}^{H}\left(\hat{\boldsymbol{\theta}}^{(k)}\right) \mathbf{A}\left(\hat{\boldsymbol{\theta}}^{(k)}\right)\right)^{-1} \mathbf{A}^{H}\left(\hat{\boldsymbol{\theta}}^{(k)}\right)$ requires approximately $2 N^{2} M+N^{3}$ operations; the calculation of the complete data $\hat{\mathbf{X}}^{(k)}$, of which the most important step is estimating the missing data $\hat{\mathbf{Y}}_{\mathbf{p}}=\mathbf{A}_{\mathbf{p}}\left(\hat{\boldsymbol{\theta}}^{(k)}\right) \hat{\mathbf{S}}^{(k)}$, is of complexity $\left(2\left(M^{\prime}-M\right) N L\right)$. The following step consists in estimating the matrix of covariance $\hat{\mathbf{R}}^{(k)}$ of the complete data $\hat{\mathbf{X}}^{(k)}$, it requires $\left(M^{\prime 2} L\right)$ operations. The eigendecomposition of $\hat{\mathbf{R}}^{(k)}$ involves $\frac{4}{3} M^{\prime 3}$ operations. Finally, the $M$-step, i.e. ESPRIT, requires the computation of the eigenvalues of the covariance matrix, which demands $O\left(N^{3}\right)$ operations. Thus, for one iteration, the number of operations required is: $O\left(2 N^{3}+2 M^{\prime} N L+M^{\prime 2} L+\frac{4}{3} M^{\prime 3}\right)+2 N^{2} M$. The predominant term is $\frac{4}{3} M^{\prime 3}$ corresponding to the eigendecomposition of $\hat{\mathbf{R}}^{(k)}$. The complexity of this expensive phase can be reduced by methods of subspaces tracking because only $N$ eigenvectors associated with the $N$ greater eigenvalues are required. This number should be multiplied by the number of iterations. In practice, the number of iterations required by EM-ESPRIT in order to converge is small (lower than 10). 


\section{Simulation results}

Several simulations were carried out in order to study different aspects of EM-ESPRIT in different conditions. Several parameters are studied: the Root-MSE (RMSE) in function of the SNR, the resolution, the convergence, the effect of the initialization and the effect of the number of sensors used in the VULA. And finally, the advantages of the NLA compared to the ULA using EMESPRIT are shown. $\Delta$ is taken as the half-wavelength. The maximal number of iterations is fixed to 30 unless otherwise indicated. The number of snapshots is 500 and 500 trials were achieved for each value. The initialization is done using the classical beamforming estimation.

\subsection{RMSE in function of SNR}

In this part, EM-ESPRIT is compared to other methods proposed in literature. The RMSE of the DOA estimates is drawn in function of the SNR using EM-ESPRIT. This study is achieved on both types of NLA. First consider the sparse array defined by the positions of its sensors: $\mathbf{d}=[0,2,5] \frac{\lambda}{2}$. Notice that this array is not a minimum redundancy linear array since it presents holes in the set of intersensor separations. The equipowered sources are located at $\left[-5^{\circ}, 10^{\circ}\right]$. The RMSE of the source at $10^{\circ}$ is plotted at Fig. 3 . The result of the second source is similar and is not represented. The corresponding CRB is also represented. Besides, EM-ESPRIT is compared to Root-MUSIC [3], the interpolation method of Friedlander [5], to EM-IQML [6] and to the method MST [8]. EM-ESPRIT presents better performance than the other methods. For the low values of the SNR, EM-ESPRIT has a smaller RMSE than Root-MUSIC, the interpolation of Friedlander and MST. EM-IQML presents a lower RMSE than EM-ESPRIT, but in the middle zone, EMESPRIT is more performant. Besides, EM-ESPRIT has a lower complexity than EM-IQML. In the asymptotic zone (high values of SNR), EM-ESPRIT has similar behavior to Root-MUSIC whereas the RMSE of the Friedlander interpolation moves away from the CRB, which is expected since the interpolation error introduced by this method is independent of the SNR. The RMSE corresponding to the MST method also moves away from the CRB because of the truncation error, knowing that a high order is chosen (21) for the truncation. This means that the rooting search in the MST method is performed on a polynomial of order (42).

The same simulation is repeated for the nonregular array. In this case, Root-MUSIC cannot be applied neither EM-IQML. Thus, EM-ESPRIT is compared to Spectral MUSIC, the Friedlander interpolation and to the method MST. The array is defined by $\mathbf{d}=[0,1.32,3.03,5] \frac{\lambda}{2}$ and the sources are the same as above located at $\left[-5^{\circ}, 10^{\circ}\right]$. The RMSE of the source at $10^{\circ}$ is represented at Fig. 4 with respect to the SNR. Fig. 4 shows the superiority of EM-ESPRIT compared to Spectral MUSIC for low SNR. It also shows that the correspondant RMSE approaches the CRB, in contrary to the Friedlander interpolation and MST that move away from the CRB because of the presence of the interpolation error and the truncation error respectively. Thus, EM-ESPRIT does not suffer from the problems encountered in other methods.

In the results simulation shown in Fig. 5, the mean behavior of EM-ESPRIT is studied not on a fixed geometry but on a set of arrays whose sensors positions are known but randomly chosen. The idea is to fix the aperture and the number of sensors of the NLA and for each trial, different sensors positions are uniformly generated (only the positions of the first and the last sensors are fixed to insure the aperture).

Consider the sparse array with an aperture of 6 and a number of sensors of $M=3$. The sources are located at $\left[-5^{\circ}, 10^{\circ}\right]$. The averaged RMSE over the whole sparse array is represented at Fig. 5 in function of the SNR. It is compared to the averaged RMSE of Root-MUSIC. EM-ESPRIT presents better performance, specially for highly noisy data. Furthermore, the averaged RMSE 


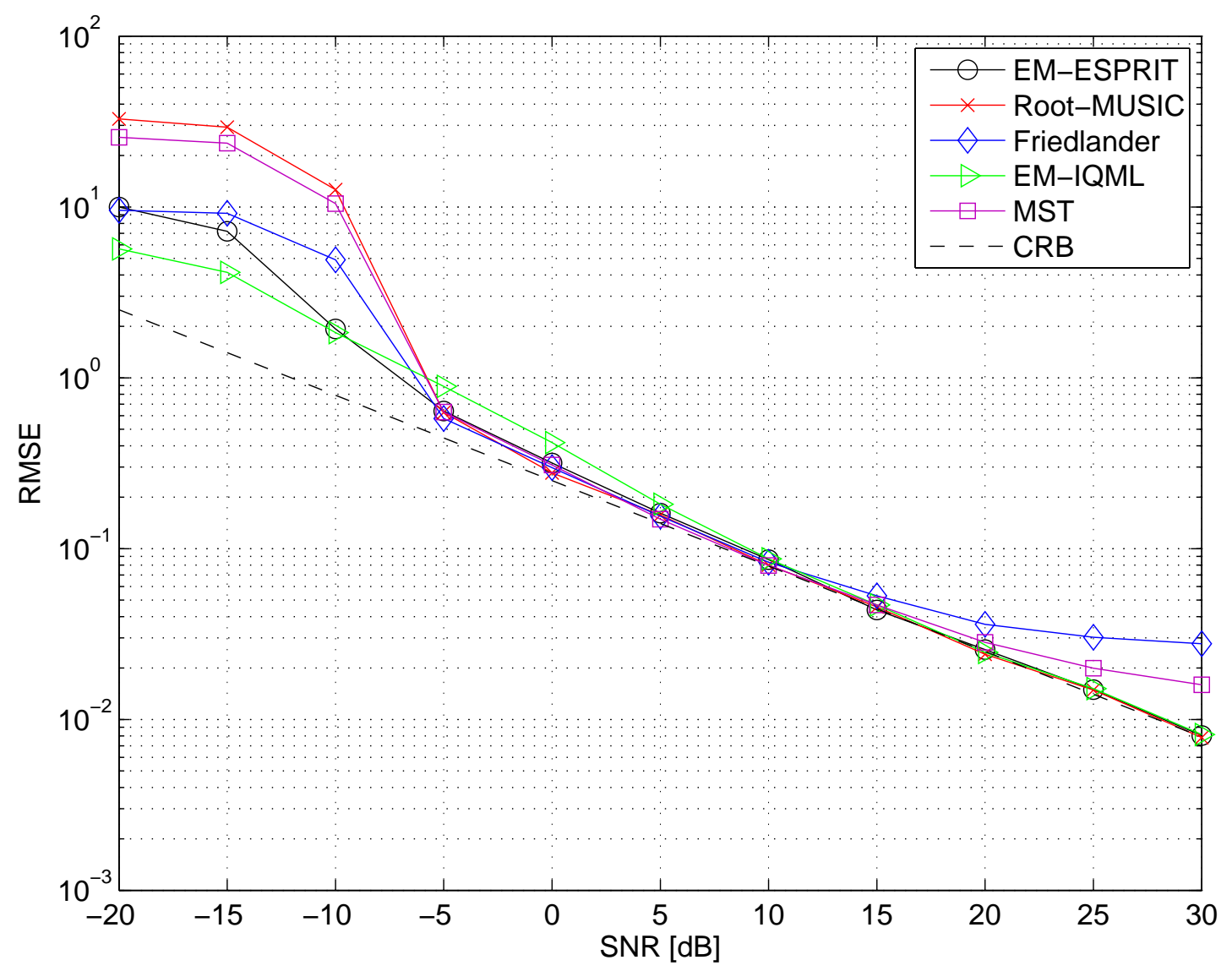

Fig. $3 \mathrm{RMSE}$ of the source at $10^{\circ}$ for the sparse array $\mathbf{d}=[0,2,5] \frac{\lambda}{2}, D O A=\left[-5^{\circ}, 10^{\circ}\right]$.

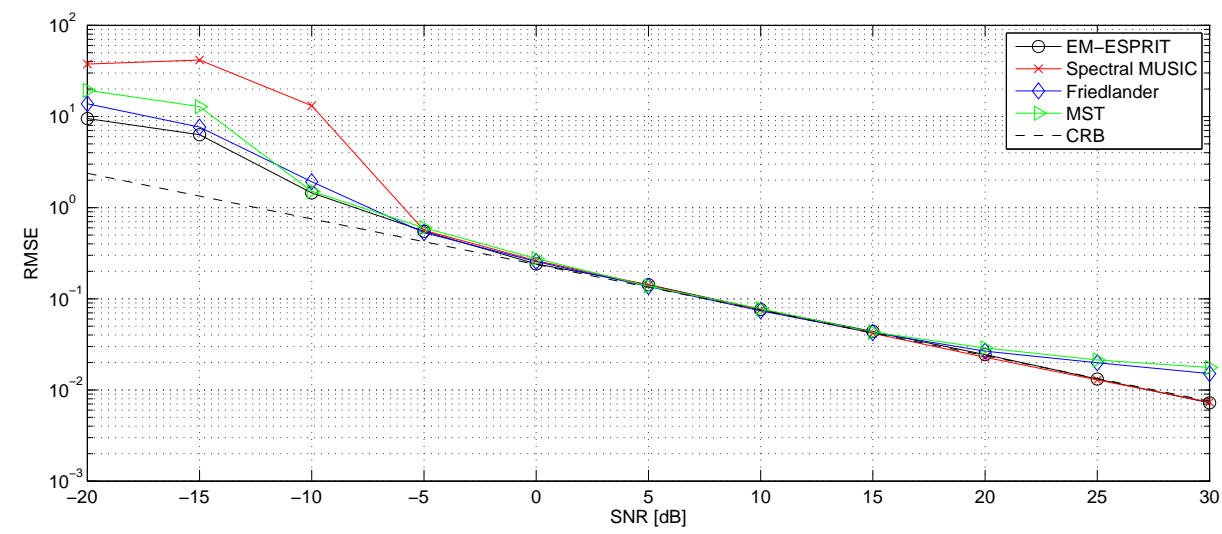

Fig. 4 RMSE of the source at $10^{\circ}$ for the nonregular array $\mathbf{d}=[0,1.32,3.03,5] \frac{\lambda}{2}, D O A=\left[-5^{\circ}, 10^{\circ}\right]$.

slightly changes from the RMSE obtained with EM-ESPRIT applied to the array $\mathbf{d}=[0,2,5] \frac{\lambda}{2}$ 


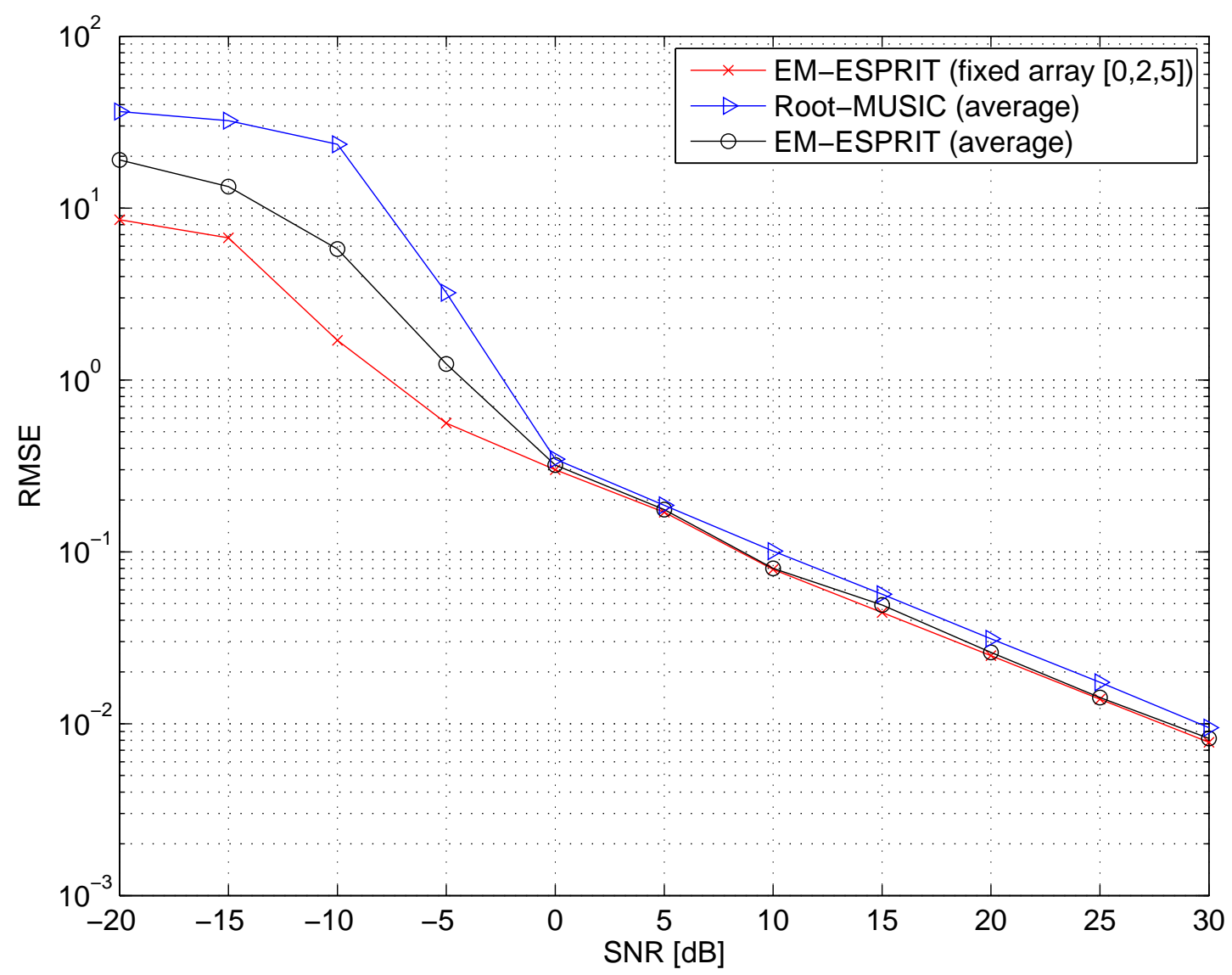

Fig. 5 RMSE for the sparse array of aperture 6 and a number of sensors of $M=3, D O A=\left[-5^{\circ}, 10^{\circ}\right]$.

considered in previous simulations. This simulation shows that EM-ESPRIT does not show any dysfunctional behavior for particular geometry cases.

At last, the performance of EM-ESPRIT is studied for a major number of sources. Consider the sparse array defined by the position of its sensors: $\mathbf{d}=[0,1,4,7,13,15,18,23] \frac{\lambda}{2}$. The number of sources is fixed to 5 , located at $\left[-10^{\circ}, 0^{\circ}, 15^{\circ}, 30^{\circ}, 50^{\circ}\right]$. EM-ESPRIT is compared to RootMUSIC and the RMSE of all the sources are represented at Fig. 6 with respect to the SNR.

This figure shows clearly the superiority of the EM-ESPRIT performance compared to RootMUSIC in the presence of an important number of sources, specially for high noise level.

\subsection{Resolution}

In this simulation, the resolution of EM-ESPRIT is considered. This is an important point, it shows whether EM-ESPRIT is capable of separating two close spaced sources. Consider the case of nonregular array with the same array used before: $\mathbf{d}=[0,1.32,3.03,5] \frac{\lambda}{2}$. The results for the sparse array case are not shown here but they are similar. Consider $N=2$ sources where the first angle is fixed to $0^{\circ}$. The second angle is separated by $\Delta \theta$ where $\Delta \theta$ increases from $1^{\circ}$ to $30^{\circ}$. The SNR is fixed to $10 \mathrm{~dB}$. The RMSE of the source at $0^{\circ}$ is represented at Fig. 7 in function of $\Delta \theta$. 


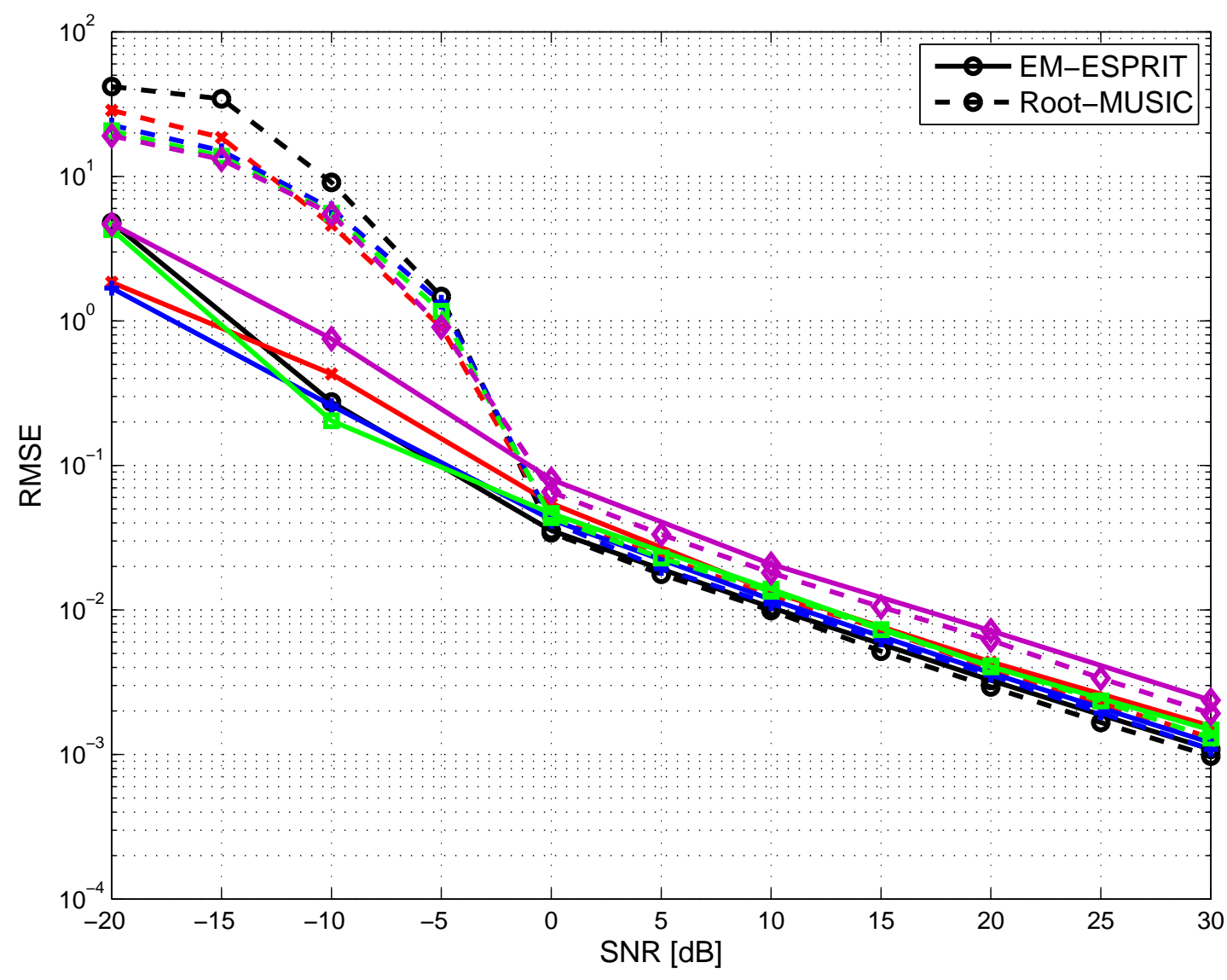

Fig. 6 RMSE in function of SNR for the sparse array $\mathbf{d}=[0,1,4,7,13,15,18,23] \frac{\lambda}{2}, \quad D O A=$ $\left[-10^{\circ}, 0^{\circ}, 15^{\circ}, 30^{\circ}, 50^{\circ}\right]$.

The resolution of EM-ESPRIT is compared to the one corresponding to Spectral MUSIC and to the Friedlander interpolation. EM-ESPRIT presents a better resolution than the other methods. Results show that for $\Delta \theta \geq 3^{\circ}$, EM-ESPRIT is the most accurate method and approaches the CRB. Furthermore, EM-ESPRIT presents better resolution than Spectral MUSIC specially for low values of $\Delta \theta^{\circ}$. It is also more accurate than the Friedlander interpolation for almost all the values of $\Delta \theta$.

\subsection{Convergence}

In this section, the convergence of the proposed method is studied. In general, the EM algorithm is reputed of slow convergence. Thus, the approximate number of iterations needed to reach the convergence is evaluated for several values of SNR.

In order to evaluate the convergence of the EM-ESPRIT algorithm, two criteria are used. The first one is the error of the estimate at each iteration, since the simulation is done only for one trial. The second one is the value of the simplified negative log-likelihood function at each iteration (since the EM algorithm maximizes the ML criterion in order to estimate the DOA). 


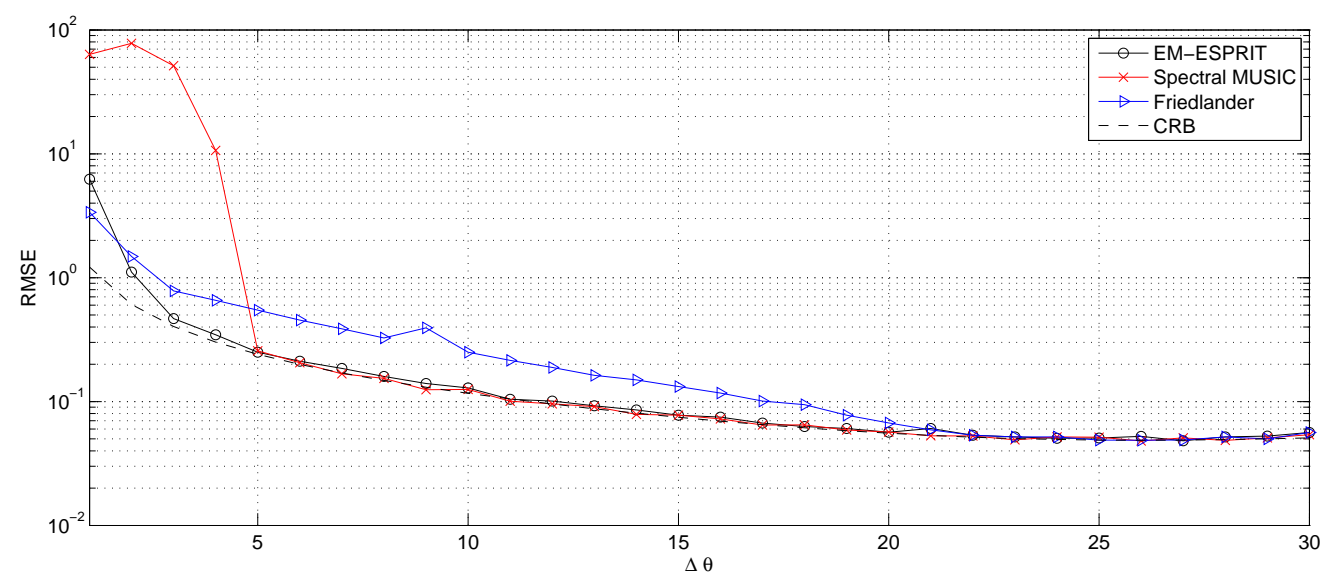

Fig. 7 Resolution of the source at $0^{\circ}$ for the nonregular array $\mathbf{d}=[0,1.32,3.03,5] \frac{\lambda}{2}, \mathrm{SNR}=10 \mathrm{~dB}, N=2$ sources.

The log-likelihood of the observed data $\mathbf{Y}$ is given by:

$$
\mathcal{L}(\Theta, \mathbf{Y})=-\frac{L M}{2} \ln \left(2 \pi \sigma^{2}\right)-\frac{1}{2 \sigma^{2}} \sum_{l=1}^{L}\|\mathbf{Y}(l)-\mathbf{A}(\theta) \mathbf{S}(l)\|^{2} .
$$

The evolution of $\mathcal{L}\left(\hat{\boldsymbol{\Theta}}^{(k)}, \mathbf{Y}\right)$ shows if the algorithm converges with the iterations. When simplifying the expression of (19), the maximization of the criterion can be reduced to the minimization of the simplified negative log-likelihood:

$$
\mathcal{L}_{1}(\Theta, \mathbf{Y})=\sum_{l=1}^{L}\|\mathbf{Y}(l)-\mathbf{A}(\theta) \mathbf{S}(l)\|^{2} .
$$

The criterion $\mathcal{L}_{1}(\Theta, \mathbf{Y})$ is used in the simulations. It should decrease with the iterations to reach the minimal value of the negative log-likelihood noted by $\mathcal{L}_{1, \min }\left(\hat{\Theta}_{M L}, \mathbf{Y}\right)$ obtained by a direct minimization of the ML function $\mathcal{L}_{1}(\Theta, \mathbf{Y})$.

Consider the same nonregular array $\mathbf{d}=[0,1.32,3.03,5] \frac{\lambda}{2}$ with the sources impinging from $\left[-5^{\circ}, 10^{\circ}\right]$. The estimation error $\left|\theta-\hat{\theta}^{(k)}\right|$ of the source at $10^{\circ}$ in function of the iterations is plotted at Fig. 8 for three SNR values: $-10,0$ and $10 \mathrm{~dB}$. The negative log-likelihood $\mathcal{L}_{1}(\Theta, \mathbf{Y})$ is also plotted at Fig. 9 for the 3 SNR values: $-10,0$ and $10 \mathrm{~dB}$, as well as the minimal value of the negative log-likelihood $\mathcal{L}_{1, \min }\left(\hat{\Theta_{M L}}, \mathbf{Y}\right)$.

Iteration 0 corresponds to the initialization value using the classical beamforming algorithm. EM-ESPRIT converges for the three SNR values. Furthermore, Fig. 8 and 9 show that the number of iterations to reach the convergence is small: for $\mathrm{SNR}=-10 \mathrm{~dB}$, it is about 10 and for $\mathrm{SNR}=0$ and 10, it is approximately 5. Thus, the convergence of EM-ESPRIT is relatively fast. In Fig. 9, the negative log-likelihood reaches the minimal value $\mathcal{L}_{1, \min }\left(\Theta_{M V}, \mathbf{Y}\right)$ for each SNR. This is an illustration of the convergence of the method.

\subsection{Initialization sensitivity}

The effect of the initial values on the results of EM-ESPRIT is considered now. It is known that the algorithm EM is sensitive to the initialization. Until now, the initial values are estimated using the beamforming algorithm, but in this situation, the initial value is fixed. In this simulation, 

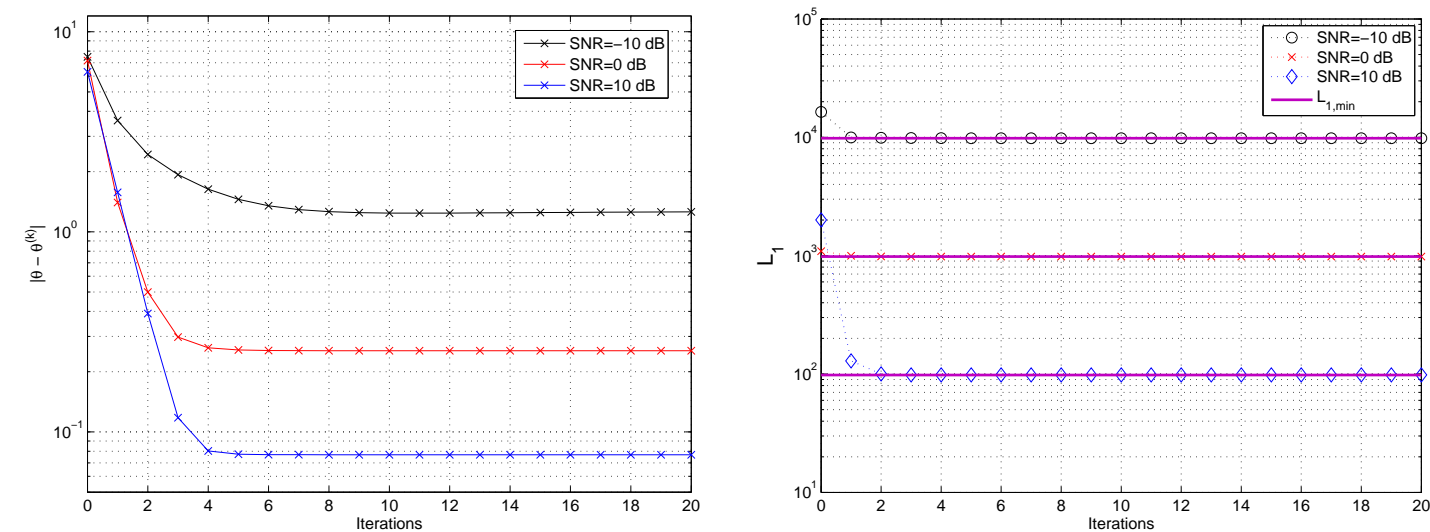

Fig. 8 RMSE of the source at $10^{\circ}$ for the nonregular Fig. 9 Negative log-likelihood for the nonregular ararray $\mathbf{d}=[0,1.32,3.03,5] \frac{\lambda}{2}$, at $\mathrm{SNR}=-10,0$ and $10 \mathrm{~dB}$, ray $\mathbf{d}=[0,1.32,3.03,5] \frac{\lambda}{2}$, at $\mathrm{SNR}=-10,0$ and $10 \mathrm{~dB}$, $D O A=\left[-5^{\circ}, 10^{\circ}\right]$. $D O A=\left[-5^{\circ}, 10^{\circ}\right]$.

consider the case of one source and study the effect of the initial value choice on the EM-ESPRIT behavior. Consider the nonregular array defined by $\mathbf{d}=[0,1.32,3.03,5] \frac{\lambda}{2}$ and the source located at $\theta_{1}=10^{\circ}$. The initial value $\theta^{(0)}$ varies inside $\left[\theta_{1}-30^{\circ}, \theta_{1}+30^{\circ}\right]$. The RMSE of the source in function of $\theta^{(0)}$ is plotted at Fig. 10 for $\mathrm{SNR}=-10$ and $10 \mathrm{~dB}$. In the case of $\mathrm{SNR}=-10 \mathrm{~dB}$ the required accuracy of the initialization is about $\pm 10^{\circ}$ so that EM-ESPRIT to converge. For SNR $=10 \mathrm{~dB}$, the initial value can be logically less accurate and the required accuracy is about $\pm 17^{\circ}$. Other simulations have shown that when the array aperture increases, the required accuracy of the initial value also increases. In practice, the initialization by the beamforming is enough for the algorithm to converge.

\subsection{Number of snapshots}

In this simulation, the effect of the number of snapshots is considered. Consider the case of nonregular array with the same array used before: $\mathbf{d}=[0,1.32,3.03,5] \frac{\lambda}{2}$. Consider $N=2$ sources where the sources are located at $\left[-5^{\circ}, 10^{\circ}\right]$. The SNR is fixed to $10 \mathrm{~dB}$. The RMSE of the source at $5^{\circ}$ is represented at Fig. 11 in function of the number of snapshots. The result of EM-ESPRIT is compared to the one corresponding to Spectral MUSIC.

It can be seen that the number of snapshots does not affect the performance of EM-ESPRIT. EM-ESPRIT approaches the CRB curve since $L=10$. Also, the result of EM-ESPRIT is better than Spectral MUSIC for small number of snapshots.

\subsection{Number of sensors of the VULA}

The effect of the choice of the number of sensors $M^{\prime}$ of the VULA in the case of nonregular array case is studied. Consider the case $M^{\prime} \geq M$. Consider the array $\mathbf{d}=[0,1.32,3.03,5] \frac{\lambda}{2}$ and the sources at $\left[-5^{\circ}, 10^{\circ}\right]$. The RMSE of the source at $-5^{\circ}$ as well as the CRB are plotted in function of the number the sensors in the VULA $M^{\prime}$ at Fig. 12 for $\mathrm{SNR}=10$ and $0 \mathrm{~dB}$. For both SNR values, it is clear that increasing the number of sensors does not provide any advantage. Therefore, our choice falls on $M^{\prime}=M$, it needs less computational time compared to larger values of $M^{\prime}$. 


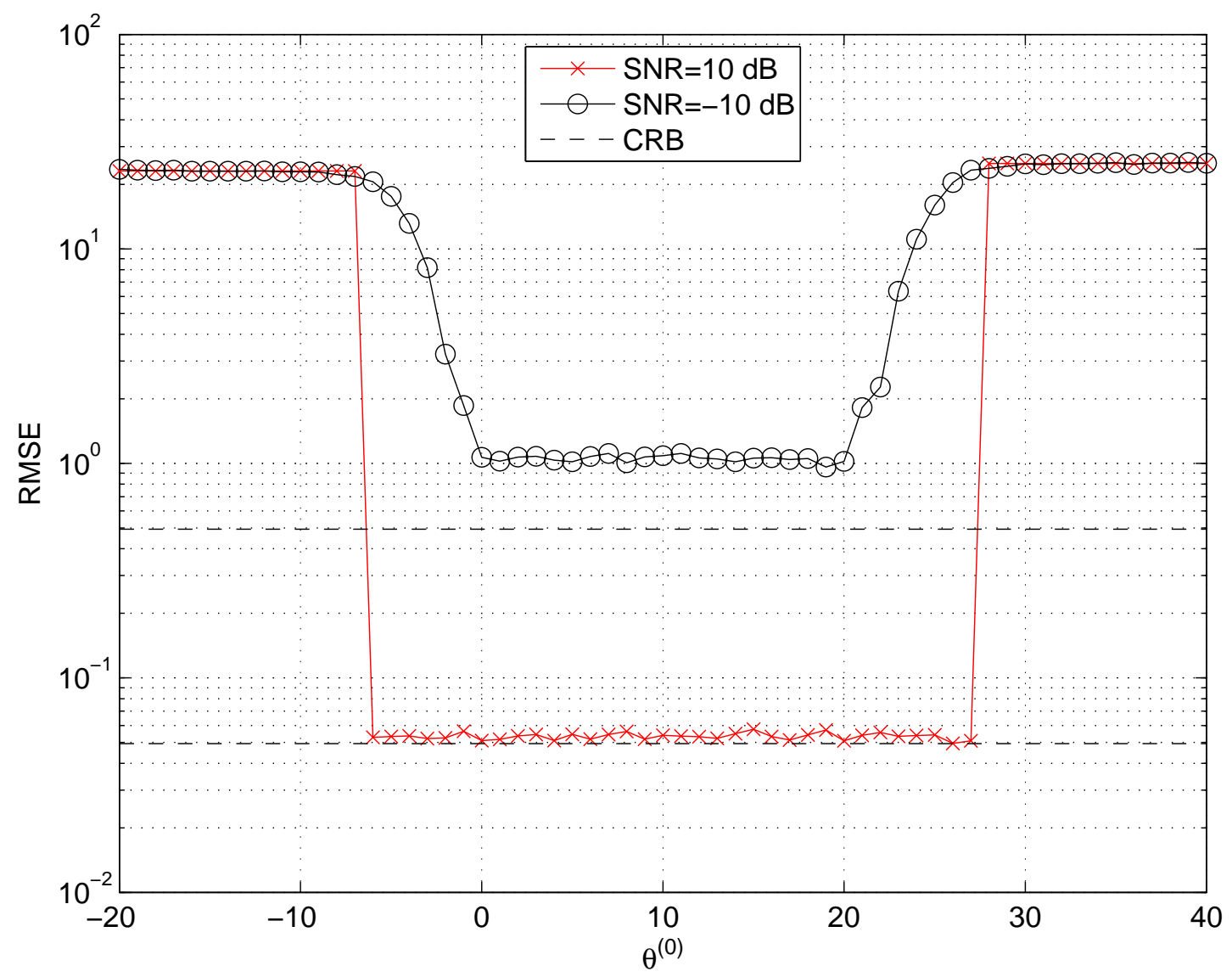

Fig. 10 RMSE of the source for the nonregular array $\mathbf{d}=[0,1.32,3.03,5] \frac{\lambda}{2}, \mathrm{SNR}=-10$ and $10 \mathrm{~dB}, N=1$ source.

\section{Application: reduction of the number of sensors}

In the introduction, it is stated that an interesting application would be to remove sensors of an array to limit the production cost without modifying the performance. In this section, the advantages of using the NLA instead of its equivalent ULA are illustrated by applying EMESPRIT. Let us denote by $\mathrm{ULA}_{M^{\prime}}$ the ULA with aperture and number of sensors equal to $M^{\prime}$ and by $\mathrm{NLA}_{M^{\prime}, M}$ an NLA with aperture $M^{\prime}$ and $M$ sensors. In [3], an analytical study was achieved in order to show the advantages of the NLA. This study was made using the CRB values on a NLA with a centro-symmetric with empty center and in the case of one source. In the case of a ULA and an NLA having the same aperture $M^{\prime}$, i.e. $\mathrm{ULA}_{M^{\prime}}$ and $\mathrm{NLA}_{M^{\prime}, M}$, the ratio of $\mathrm{CRB}$ correspondant to the arrays is given by:

$$
\frac{C R B_{U L A_{M^{\prime}}}}{C R B_{N L A_{M^{\prime}, M}}} \approx 1-\left(\frac{\delta}{M^{\prime}}\right)^{3}
$$

where $\delta=M^{\prime}-M$ is the number of missing sensors in the NLA. If the number of missing sensors $\delta$ is small enough with respect to $M^{\prime}$, the last term in (21) is negligible. Thus, the NLA performance is almost the same as the ULA having the same aperture. Now if a ULA and an 


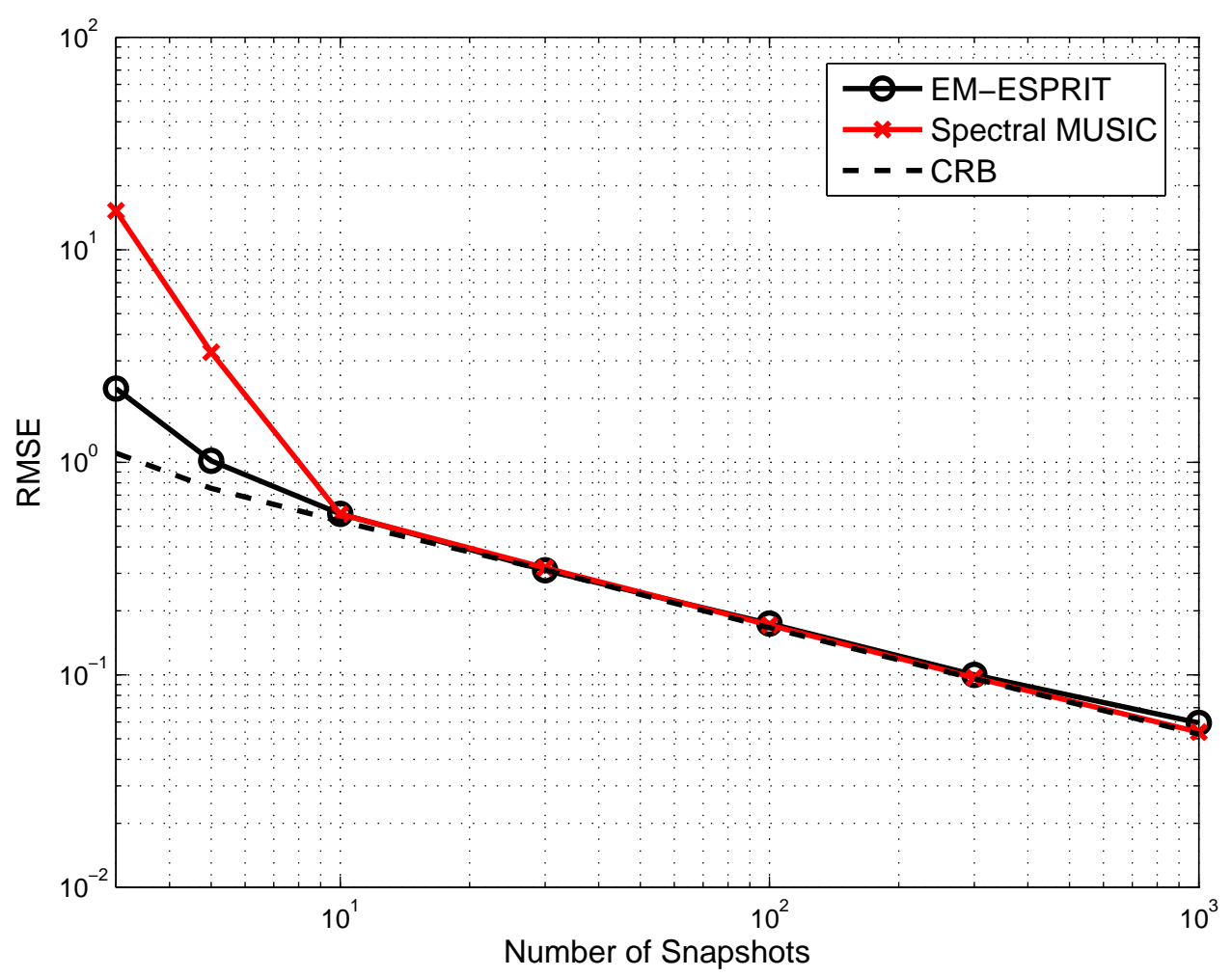

Fig. 11 Effect of the number of snapshots for the nonregular array $\mathbf{d}=[0,1.32,3.03,5] \frac{\lambda}{2}, \mathrm{SNR}=10 \mathrm{~dB}, N=2$ sources.

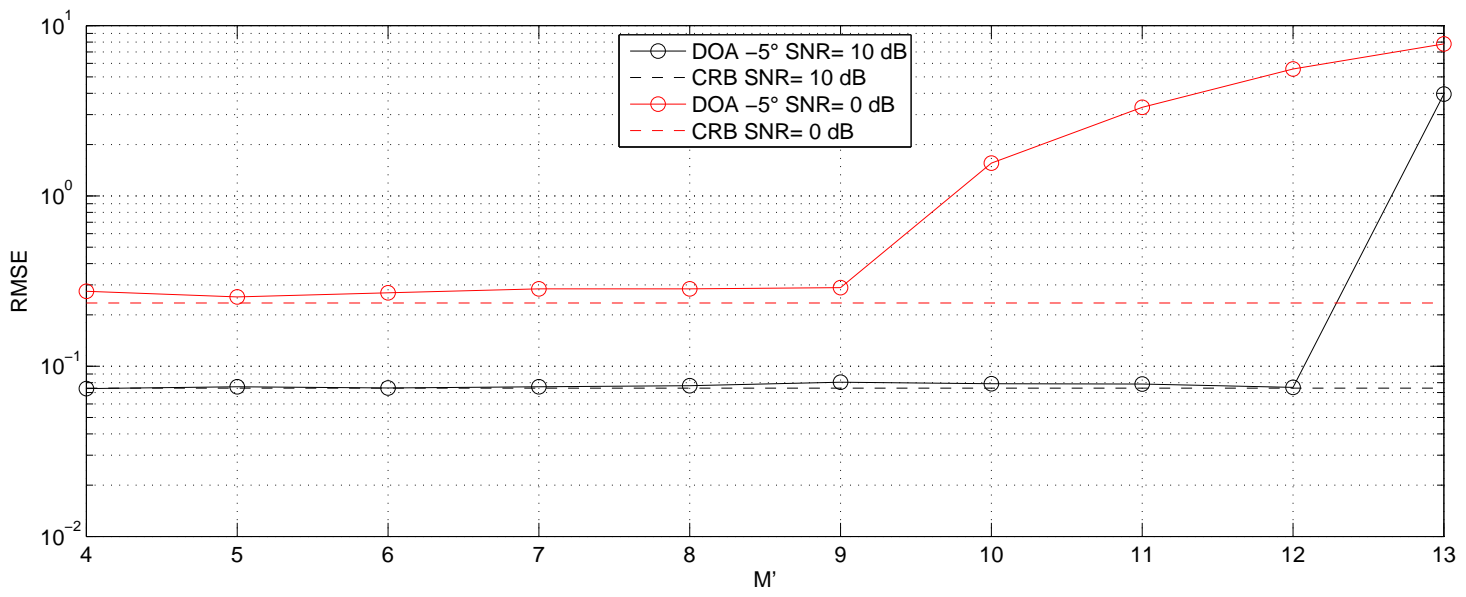

Fig. 12 RMSE of the source at $-5^{\circ}$ for the nonregular array $\mathbf{d}=[0,1.32,3.03,5] \frac{\lambda}{2}, \mathrm{SNR}=10$ and $0 \mathrm{~dB}, D O A=$ $\left[-5^{\circ}, 10^{\circ}\right]$.

NLA having the same number of sensors are considered, i.e. $\mathrm{ULA}_{M}$ and $\mathrm{NLA}_{M^{\prime}, M}$, the ratio of 
the CRB becomes:

$$
\frac{C R B_{U L A_{M}}}{C R B_{N L A_{M^{\prime}, M}}}=1+3\left(\frac{M^{\prime}}{M}\right)^{2}-3 \frac{M^{\prime}}{M}>1 .
$$

Thus, the NLA with a bigger aperture and the same number of sensors as the ULA presents better performance.

In this section, the case of two sources is considered. Thus, the comparison cannot be made on the CRB values, it is done with respect to the RMSE of the EM-ESPRIT algorithm. Consider a ULA 10 with $M^{\prime}=10$ sensors spaced by a half-wavelength. The NLA are created by eliminating the sensors successively, one after another. Similar results can be obtained when choosing other structures. The chosen NLA are given in table 1 . Notice that all the $\mathrm{NLA}_{10, M}$ have the same

\begin{tabular}{|c|c|c|c|c|c|c|}
\hline \multirow{2}{*}{$\begin{array}{c}\text { Number } \\
\text { of sensors } \\
(M)\end{array}$} & \multicolumn{3}{|c|}{ NLA } & \multicolumn{3}{|c|}{ ULA } \\
\hline & Array & $\begin{array}{c}\text { Aperture } \\
\left(M^{\prime}\right)\end{array}$ & $\begin{array}{c}\text { Positions }[\lambda / 2] \\
\text { (d) }\end{array}$ & Array & $\begin{array}{c}\text { Aperture } \\
\left(M^{\prime}\right)\end{array}$ & $\begin{array}{c}\text { Positions }[\lambda / 2] \\
\text { (d) }\end{array}$ \\
\hline 10 & - & - & - & $\mathrm{ULA}_{10}$ & 10 & {$\left[\begin{array}{llllllllll}0 & 1 & 2 & 3 & 4 & 5 & 6 & 7 & 8 & 9\end{array}\right]$} \\
\hline 9 & $\operatorname{NLA}_{10,9}$ & 10 & {$\left[\begin{array}{lllllllll}0 & 1 & 2 & 3 & 4 & 6 & 7 & 8 & 9\end{array}\right]$} & $\mathrm{ULA}_{9}$ & 9 & {$\left[\begin{array}{lllllllll}0 & 1 & 2 & 3 & 4 & 5 & 6 & 7 & 8\end{array}\right]$} \\
\hline 8 & $\mathrm{NLA}_{10,8}$ & 10 & {$\left[\begin{array}{llllllll}0 & 1 & 2 & 4 & 5 & 7 & 8 & 9\end{array}\right]$} & $\mathrm{ULA}_{8}$ & 8 & {$\left[\begin{array}{llllllll}0 & 1 & 2 & 3 & 4 & 5 & 6 & 7\end{array}\right]$} \\
\hline 7 & $\mathrm{NLA}_{10,7}$ & 10 & {$\left[\begin{array}{lllllll}0 & 1 & 2 & 4 & 5 & 8 & 9\end{array}\right]$} & $\mathrm{ULA}_{7}$ & 7 & {$\left[\begin{array}{lllllll}0 & 1 & 2 & 3 & 4 & 5 & 6\end{array}\right]$} \\
\hline 6 & $\operatorname{NLA}_{10,6}$ & 10 & {$\left[\begin{array}{llllll}0 & 2 & 4 & 5 & 8 & 9\end{array}\right]$} & $\mathrm{ULA}_{6}$ & 6 & {$\left[\begin{array}{llllll}0 & 1 & 2 & 3 & 4 & 5\end{array}\right]$} \\
\hline 5 & $\operatorname{NLA}_{10,5}$ & 10 & {$\left[\begin{array}{lllll}0 & 1 & 4 & 8 & 9\end{array}\right]$} & $\mathrm{ULA}_{5}$ & 5 & {$\left[\begin{array}{lllll}0 & 1 & 2 & 3 & 4\end{array}\right]$} \\
\hline 4 & $\operatorname{NLA}_{10,4}$ & 10 & {$\left[\begin{array}{llll}0 & 5 & 8 & 9\end{array}\right]$} & $\mathrm{ULA}_{4}$ & 4 & {$\left[\begin{array}{llll}0 & 1 & 2 & 3\end{array}\right]$} \\
\hline 3 & $\operatorname{NLA}_{10,3}$ & 10 & {$\left[\begin{array}{lll}0 & 4 & 9\end{array}\right]$} & $\mathrm{ULA}_{3}$ & 3 & {$\left[\begin{array}{lll}0 & 1 & 2\end{array}\right]$} \\
\hline
\end{tabular}

Table 1 The NLA and ULA geometries used in the simulations.

aperture as the original $\mathrm{ULA}_{10}$ with 10 sensors. The DOA are located at $\left[-5^{\circ}, 10^{\circ}\right]$. The EMESPRIT method is applied in order to estimate the DOA in the case of NLA and ESPRIT in the case of ULA. The SNR is fixed at $10 \mathrm{~dB}$ and the RMSE is plotted versus the number of sensors. Fig. 13 shows the performance of the NLA defined previously and the ULA, for the source located at $0^{\circ}$. The results for the source at $10^{\circ}$ are similar. For each value of $M$, the performance of the arrays given by the corresponding row in table 1 is studied. Fig. 13, shows that $\mathrm{NLA}_{M^{\prime}, M}$ provides better performance than the $\mathrm{ULA}_{M}$ with the same number of sensors. Indeed, using an $\mathrm{NLA}_{10,5}$ with $M=5$ sensors and with an aperture of 10 gives better results than using a ULA 5 with 5 sensors. Thus, this example shows the importance of the aperture of the array and that EM-ESPRIT efficiently exploits the larger aperture of NLA with respect to ULA. Furthermore, focusing on the $\mathrm{NLA}_{10, M}$ curve shows that going from $M=9$ to $M=5$ slightly changes the performance. This means that instead of using 10 sensors, similar RMSE can be achieved using only half of the number of sensors, thanks to EM-ESPRIT. Thus, the NLA may have numerous gaps without affecting the RMSE performance. In conclusion, this example shows the advantages of using the NLA instead of the equivalent ULA, it needs less production cost since the performance of an NLA having the same aperture and having only half of the number of sensors of the ULA slightly changes.

\section{Conclusion}

In this paper, a new iterative method named EM-ESPRIT for DOA estimation is proposed. The key idea of EM-ESPRIT is to interpolate the existing data using the signal model in order to form a VULA and to use the ESPRIT algorithm to estimate the DOA. Another important novelty proposed in this paper is to extend this approach to all types of nonuniform arrays, where other 


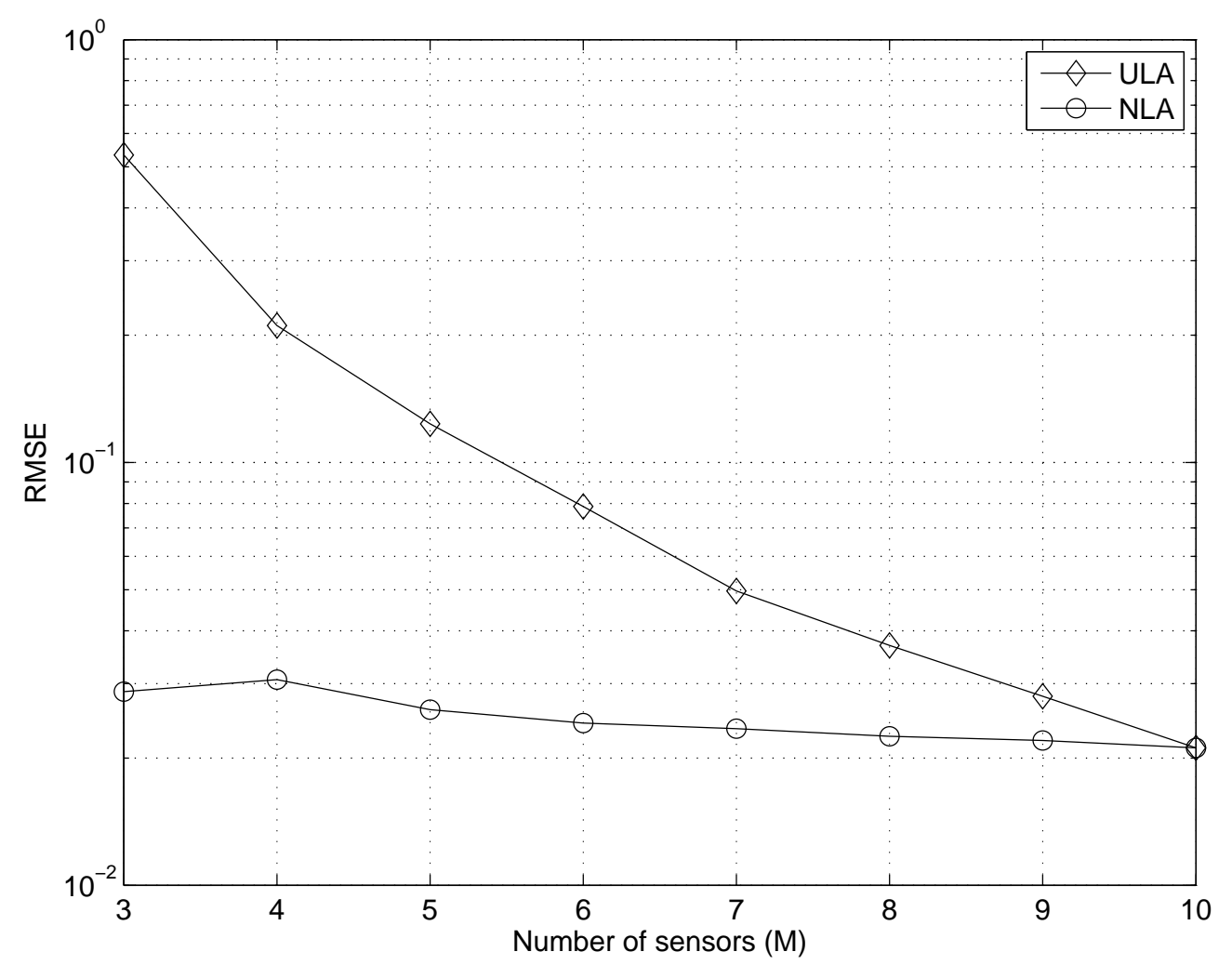

Fig. 13 RMSE for the source at $-5^{\circ}$ with $M=3,4, \ldots, 10, \mathrm{SNR}=10 \mathrm{~dB}, \mathrm{DOA}=\left[-5^{\circ}, 10^{\circ}\right]$ in the case of ULA and NLA.

methods fail to treat the nonregular case. Simulation results show that EM-ESPRIT is more robust to additive noise than the other methods with lower computational cost. Furthermore, EM-ESPRIT can fully exploit the advantages of using an NLA instead of a ULA, for instance it reduces the cost of antenna by reducing the number of sensors. In fact, using an NLA with a reasonable less number of sensors than the equivalent ULA preserves almost the same variance for DOA estimation. Another advantage is that for the same number of sensors, the NLA presents better performance since the aperture is bigger, which means that the resolution is better.

\section{References}

1. M. Zhang, Z. Zhi, Circuits systems signal process 15, no. 1, 137 (1996)

2. Z. Saric, D. Kukolj, N. Teslic, Circuits systems signal process 29, 837 (2010)

3. C. El Kassis, J. Picheral, C. Mokbel, Signal Processing 90, Issue 2, 689 (February 2010)

4. R.O. Schmidt, A signal subspace approach to multiple emitter location and spectral estimation. Ph.D. thesis, Dept. Elect. Eng., Stanford Univ., Stanford, CA (1981)

5. B. Friedlander, Signal Process. 30, no. 1, 15 (January 1993)

6. A.J. Weiss, A.S. Willsky, B.C. Levy, IEEE Trans. Aerosp. Electron. Syst. 25, no. 1, 48 (January 1989)

7. Y.I. Abramovich, D.A. Gray, A.Y. Gorokhov, N.K. Spencer, IEEE Trans. Signal Processing 46, no. 9, 2458 (September 1998)

8. F. Belloni, A. Richter, V. Koivunen, IEEE Trans. Signal Processing 55, no. 10, 4800 (October 2007)

9. M. Rübsamen, A.B. Gershman, IEEE International Conference on Acoustics, Speech and Signal Processing Issue, March 31 2008-April 4 2008, 2317 (2008)

10. Y.H. Chen, Y.S. Lin, IEEE Antennas and Propagation Society International Symposium 3, 1576 (1994) 
11. R. Roy, T. Kailath, IEEE Transactions on Acoutics, Speech, and Signal Processing 37, 984 (1989)

12. A.J. Barabell, Proc. ICASSP, Boston, M.A. pp. 336-339 (1983)

13. A.P. Dempster, N.M. Laird, D.B. Rubin, Royal Statistical Society, series B 39, 1 (1977)

14. B. Ottersten, M. Viberg, T. Kailath, IEEE Trans. on Signal Processing 39, no. 5, 1122 (May 1991)

15. M. Feder, E. Weinstein, IEEE Trans. Acoust., Speech, Signal Processing 36, no. 4, 477 (April 1988) 